\title{
Computational aircraft dynamics and loads
}

\author{
$\mathrm{K}_{\text {APPA }}{ }^{1}$ and J ARGYRIS ${ }^{2}$ \\ ${ }^{1}$ Northrop Corporation, Aircraft Division, MS 3969/63, One Northrop \\ Avenue, Hawthorne, California 90250-3277, USA \\ ${ }^{2}$ Institute for Computer Applications, Pfaffenwaldring 27 D-70569 \\ Stuttgart, University of Stuttgart, Germany
}

\begin{abstract}
An alternative approach that has the potential to advance classical methods of flight load prediction by combining computational fluid dynamics (CFD), structural flexibility and the interaction of flight control system (FCS) in a multidisciplinary analysis package is described. The method employs the concept of system identification to characterize aircraft dynamics in the state space coordinate system and includes an adaptive control law design methodology. An extended account of the theoretical basis for the new multidisciplinary flight manoeuvre analysis will be presented in one of a seven-volume series on computational mechanics by Argyris and his associates to be published shortly. However, as a precursor to the complete work, a brief account of the theoretical development leading to this loads prediction methodology is included in this paper.
\end{abstract}

Keywords. Flight loads; manoeuvre loads; multidisciplinary computational methods; external loads.

\section{Introduction}

The structural design of aircraft requires the knowledge of external loads acting on individual components. The computation of such loads depends on the flight environment to which a particular aircraft is subjected. For example, commercial (or transport) aircraft is expected to withstand loads due to level flight, gust encounter, landing and take off, and ground handling. On the other hand, a combat aircraft is required to sustain additional loads due to rapid manoeuvres which are several times higher than normal operational loads. The development of load spectra and load envelope depends on a particular aircraft's mission and is typically specified by the procuring agencies.

The critical design flight conditions used to compute load exceedance envelopes such as shear vs. bending moment, and torsion vs. bending moment can be simulated by moving the control sticks at desired rates (see Appa 1991). The modern aircraft design methodology requires that the flight control systems (FCS) be included in 


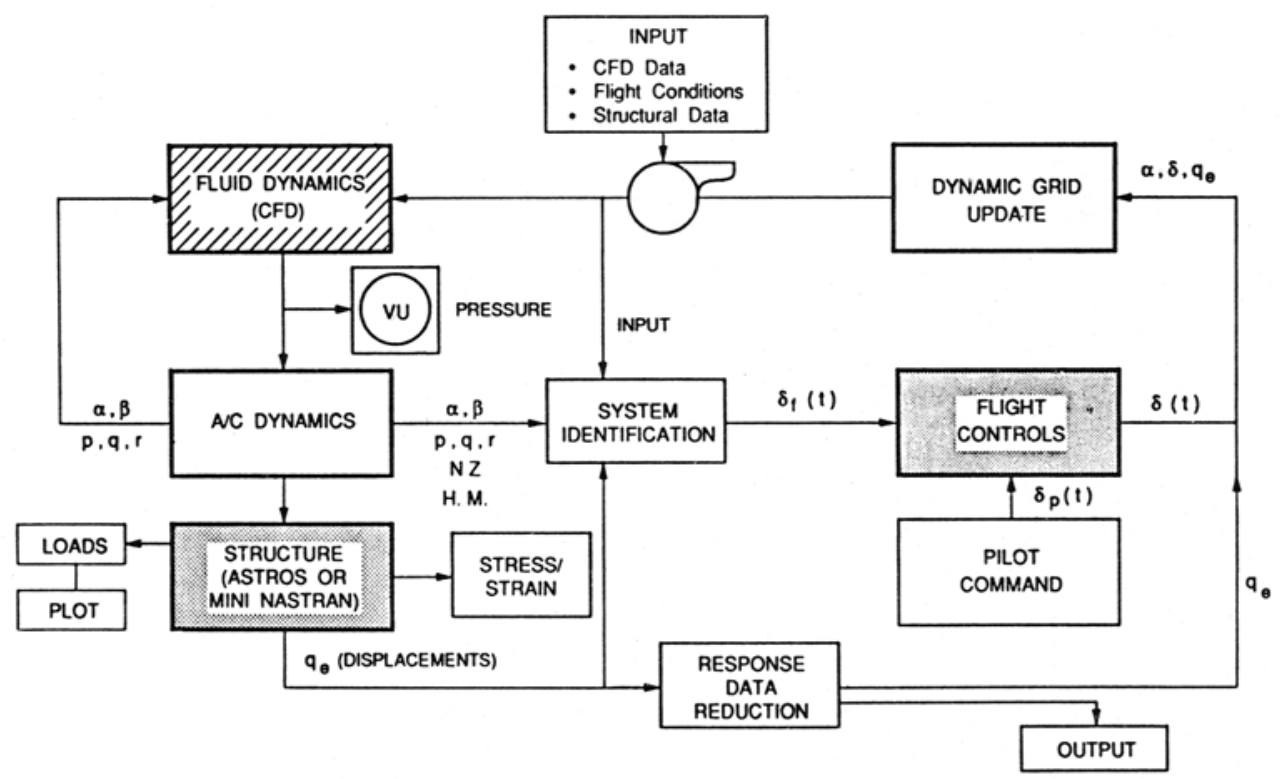

Figure 1. A schematic diagram of computational aircraft dynamics and loads.

analysis and design cycles so that effective use of the control surfaces may be made to predict the design loads while optimizing aircraft stability, control and performance characteristics. Therefore, the proposed loads analysis method integrates an on-line control law design methodology combining aircraft flexibility and nonlinear aerodynamic forces computed by the CFD methods.

The overall concept of this multidisciplinary analysis approach is depicted in figure 1. The nonlinear aerodynamic forces will be computed by the CFD module. The transient response of the aircraft subject to control stick input will be determined in the aircraft dynamics module. The input and response characteristics data will be analysed in the system identification module to derive the state space matrices. The state space analysis is then employed; (1) to design adaptive flight control laws, (2) to perform flight manoeuvres, and (3) to compute net design loads. The test cases considered in this study demonstrate the computational feasibility of flight manoeuvre analysis using the CFD methods.

Subsequent sections include brief descriptions of: (1) state space formulation of equations of motion of flexible aircraft, (2) implicit acceleration of the CFD method used in the present study, (3) the concept of system identification, and (4) adaptive control law design. A few test cases are included to demonstrate the overall concept of manoeuvre loads analysis.

\section{Equations of motion of flexible aircraft}

The equations of motion of an aircraft may be expressed in any convenient coordinate system. A common practice in flight mechanics is to use a body-fixed coordinate system oriented either along the body axis or the wind axis. Figure 2 shows various axes systems in which the subscripts $I, W$ and $B$ denote inertial, wind and body-fixed reference coordinates, respectively. The climb angle, $\gamma$, is denoted by the angle between 
(a)

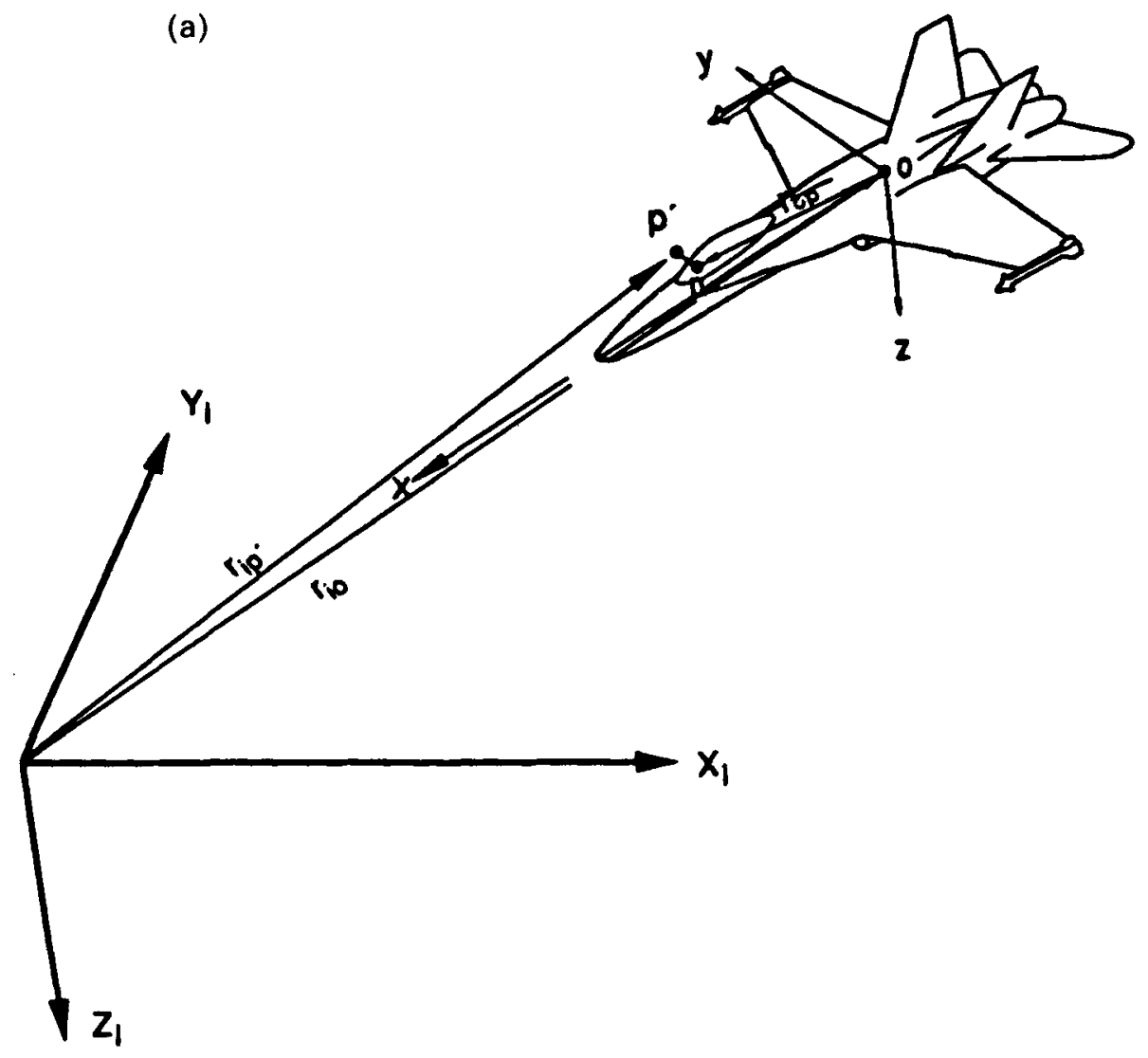

(b)

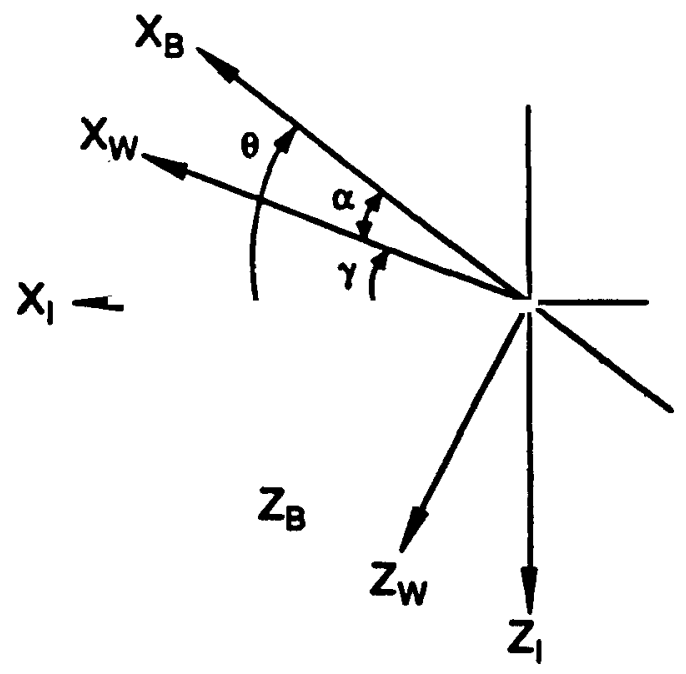

Figure 2. Reference axis systems. (a) Body-fixed coordinate system; (b) definition of aerodynamic angles (step input). 
the wind axis and the inertial frame of reference, while the aerodynamic angles $(\alpha, \beta)$ are denoted by the incidence angles between the wind and the body axes.

A system of equations of motion of a flexible aircraft in the inertial axis system is given by

$$
\mathbf{K r}+\mathbf{M r}+\mathbf{F}(\dot{\mathbf{r}}, \ddot{\mathbf{r}}, t)=0
$$

where $\mathbf{r}$ is a displacement vector, $\mathbf{K}$ is a stiffness matrix, $\mathbf{M}$ is a mass matrix and $\mathbf{F}$ is a vector of aerodynamic and other external forces. The computation of stiffness and mass matrices has by now become a routine procedure in finite element methods (FEM). This generalized analysis procedure in structural mechanics finds its origin in Argyris' work (Argyris \& Dunne 1947, 1949; Argyris 1953; Argyris \& Kelsey 1960). Hence, there is no need for further discussions regarding the computation of stiffness and mass matrices. On the other hand, the computational aspects of the nonlinear aerodynamics using CFD methods have not yet reached the degree of maturity which has been achieved by the community of structural engineers. Nevertheless, efforts are being made at an ever increasing stride to achieve that goal. We will touch upon this topic a little later.

Equation (1) represents several hundred or even thousands of degrees of freedom. But, if the structure is linear it may be replaced by a fewer modal degrees of freedom, $\boldsymbol{\eta}=\left\{\boldsymbol{\eta}_{\boldsymbol{r}}, \boldsymbol{\eta}_{\boldsymbol{e}}\right\}$, in which the displacement vector $\mathbf{r}$ will be represented by a linear combination of rigid body modes, $\boldsymbol{\Phi}_{\mathrm{r}}$, and the vibration modes, $\boldsymbol{\Phi}_{\mathrm{e}}$. Then, the generalized system of equations may be given by (Appa 1991; Argyris \& Mlejnek 1991; Argyris et al 1995)

$$
\overline{\mathbf{K}} \boldsymbol{\eta}+\overline{\mathbf{C}} \dot{\boldsymbol{\eta}}+\overline{\mathbf{M}} \ddot{\boldsymbol{\eta}}+\overline{\mathbf{F}}(\dot{\boldsymbol{\eta}}, \ddot{\boldsymbol{\eta}}, t)=0 .
$$

This is a second-order differential equation in time domain. But one can reduce this to a first-order system by replacing the independent variables by

$$
\begin{aligned}
& \mathbf{x}=\{\boldsymbol{\eta} \dot{\boldsymbol{\eta}}\}, \\
& \dot{\mathbf{x}}=\mathbf{A} \mathbf{x}+\mathbf{B u}=\mathbf{F}(\mathbf{x}, \mathbf{u}, t) .
\end{aligned}
$$

The output or the measured signals of the plant may be expressed as a linear combination of $\mathbf{x}$ and $\dot{\mathbf{x}}$, i.e.

$$
\mathbf{z}=\mathbf{H}_{1} \mathbf{x}+\mathbf{H}_{2} \dot{\mathbf{x}}=\mathbf{C x}+\mathrm{Du}
$$

where $\mathbf{u}$ denotes the control surface motion, and $\mathbf{C}$ and $\mathbf{D}$ are the measurement matrices.

The system of equations (4) can be integrated analytically provided the eigenvalues of the state matrix A lie in the left half of the $s(=\sigma+i \omega)$ plane with $\sigma<0$. Otherwise, there is a need to find a feedback gain matrix which helps to shift the roots from the right half to the left half of the $s$-plane. We will discuss this issue in subsequent sections.

\section{Computational aerodynamics: CFD}

In the last two decades highly competitive numerical schemes have been developed to predict the nonlinear pressure distributions on complex configurations. Hirsch, in 
his text books on computational fluid dynamics, discusses a number of numerical schemes developed to date and gives an extensive list of references (Hirsch 1990). These numerical schemes may broadly be classified as those based on structured or unstructured grid models. The finite difference schemes are well suited for structured grid models, while the finite element and the finite volume schemes are applicable for either model. The latter schemes show some potential for generalization of the computational algorithm such as the one used in structural mechanics. Hence, in the present study we have chosen a finite element-based CFD scheme (Argyris et al 1989).

The Euler equations of fluid motion, using the notations of Argyris et al (1989), may be written as

where

$$
\frac{\partial \mathbf{V}}{\partial t}+\sum_{i} \frac{\partial \mathbf{f}_{i}}{\partial \mathbf{x}_{i}}=0, \quad i=1,2,3
$$

$$
\mathbf{V}=\left\{\rho, \rho u_{1}, \rho u_{2}, \rho u_{3}, \rho \varepsilon\right\}
$$

is a vector of independent variables and the flux in the $i$ th coordinate direction is

$$
\mathbf{f}_{i}=u_{i} \mathbf{V}+\mathbf{e}_{i+1} p+\mathbf{e}_{\mathrm{s}} u_{i} p, \quad i=1,2,3,
$$

in which, for example, $\mathbf{e}_{2}$ denotes

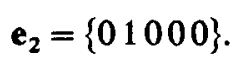

The gradient of the flux may be written as

$$
\frac{\partial \mathbf{f}_{i}}{\partial x_{i}}=\frac{\partial \mathbf{f}_{i}}{\partial \mathbf{V}} \frac{\partial \mathbf{V}}{\partial x_{i}}=\mathbf{A}_{i} \frac{\partial \mathbf{V}}{\partial x_{i}}
$$

where $A_{i}$ is the Jacobian of the flux, $\mathbf{f}_{i}$, in the coordinate direction $x_{i}$. The eigenvalues of this matrix are given by

$$
\begin{aligned}
& \lambda_{k}^{i}=u_{i}, \quad k=1,2,3 \text { (for the entropy wave), } \\
& \lambda_{4}^{i}=u_{i}+a, \quad \text { (for the forward pressure wave), } \\
& \lambda_{5}^{i}=u_{i}-a, \quad \text { (for the backward pressure wave). }
\end{aligned}
$$

The signs of these eigenvalues denote the direction in which a specific wave moves. Hence, the gradient of the flux $f_{i}$, must be evaluated in the upwind direction. Further, the Jacobian matrix $\mathbf{A}_{i}$, may be expressed in terms of its eigenvalues and eigenvectors as

$$
\mathbf{A}_{i}=\mathbf{P}_{i}\left(\Lambda_{i}^{+}+\Lambda_{i}^{-}\right) \mathbf{P}_{i}^{-1}=\mathbf{A}_{i}^{+}+\mathbf{A}_{i}^{-}
$$

where $\Lambda_{i}^{+}$and $\Lambda_{i}^{-}$denote diagonal matrices consisting of positive and negative eigenvalues, respectively, and $\mathbf{P}_{i}$ is a square matrix of eigenvectors of $\mathbf{A}_{i}$. Then from (10) assuming that $\mathbf{A}_{i}$ is constant within an element, we obtain

$$
\frac{\partial \mathbf{f}_{i}}{\partial x_{i}}=\mathbf{A}_{i}^{+} \frac{\partial^{-} \mathbf{V}}{\partial x_{i}}+\mathbf{A}_{i}^{-} \frac{\partial^{+} \mathbf{V}}{\partial x_{i}}
$$


where $\left(\partial^{+} / \partial x_{i}\right)()$ and $\left(\partial^{-} / \partial x_{i}\right)()$ denote upwind differencing depending on the sign of the eigenvalue $\lambda_{i}$.

But we know that the finite element schemes (also finite volume schemes) permit only centre differencing. Hence, it is necessary to express the upwind difference operators in terms of the centre difference operators. This can be achieved by the use of the Taylor series expansion. Thus, using the first two terms in series we obtain

$$
\begin{array}{ll}
\frac{\partial^{+} \mathbf{V}}{\partial x_{i}}=\frac{\partial \mathbf{V}}{\partial x_{i}}+\sigma \delta_{i}\left(\frac{\partial \mathbf{V}}{\partial x_{i}}\right)+\frac{1}{2} \sigma^{2} \delta_{i}^{2}\left(\frac{\partial \mathbf{V}}{\partial x_{i}}\right), & 0<\sigma<1, \\
\frac{\partial^{-} \mathbf{V}}{\partial x_{i}}=\frac{\partial \mathbf{V}}{\partial x_{i}}-\sigma \delta_{i}\left(\frac{\partial \mathbf{V}}{\partial x_{i}}\right)+\frac{1}{2} \sigma^{2} \delta_{i}^{2}\left(\frac{\partial \mathbf{V}}{\partial x_{i}}\right), & 0<\sigma<1,
\end{array}
$$

where $\delta_{i}$ ( ) and $\delta_{i}^{2}$ ( ) denote undivided first- and second-order difference operators. Substituting (16) into (15), and simplifying the algebra, we get

$$
\partial \mathbf{f}_{i} / \partial x_{i}=\partial \mathbf{f}_{i}^{* *} / \partial x_{i}
$$

where the upwind flux, $f_{i}^{* *}$, is given by

$$
\begin{aligned}
\mathbf{f}_{i}^{* *} & =\mathbf{f}_{i}^{*}\left(\mathbf{V}_{i}^{*}\right)-\sigma\left|\mathbf{A}_{i}\right| \delta_{i} \mathbf{V} \\
\mathbf{V}_{i}^{*} & =\mathbf{V}+\frac{1}{2} \sigma^{2} \delta_{i}^{2} \mathbf{V} \\
\left|\mathbf{A}_{i}\right| & =\mathbf{P}_{i}\left(\Lambda_{i}^{+}+\left|\Lambda_{i}^{-}\right|\right) \mathbf{P}_{i}^{-1}
\end{aligned}
$$

Thus, we require only central differencing which can be accomplished in the finite element scheme. The original ICA-CFD code has been modified to compute the upwind flux gradients. This eliminates the need for the artificial viscosity terms, which are arbitrary and undesirable in unsteady aerodynamic analysis consisting of low and high frequency spectra. Additional discussions relevant to the numerical computation of the upwind flux may be found in Argyris et al (1995).

\subsection{Moving boundary conditions}

The solid boundary may undergo a set of rigid body motions. $\boldsymbol{\eta}_{\mathbf{r}}$, and elastic motions, $\eta_{e}$, as discussed earlier. The rigid body motions contribute to aerodynamic attitudes resulting from lateral motions $(\dot{x}, \dot{y}, \dot{z})$ (e.g. angle of attack, $\alpha$, and sideslip, $\beta$ ) and angular rates $(\dot{\phi}, \dot{\theta}, \dot{\psi})$ (roll rate, $p$, pitch rate, $q$, and yaw rate, $r$ ). These are the relative velocities with respect to the infinite volume of fluid. Hence, the flow variables at all points must be updated. Thus, at each fluid node, $n$, we get

where

$$
\mathbf{v}^{\prime}=\mathbf{T}(\Delta \alpha, \Delta \beta) \mathbf{v}+\left[\Phi_{\mathbf{r}}\right]_{n} \boldsymbol{\theta},
$$

$\mathrm{v}=\left\{u_{1}, u_{2}, u_{3}\right\}$ are nodal velocities,

$\boldsymbol{\theta}=\{\mathrm{d} p, \mathrm{~d} q, \mathrm{~d} r\}$ are incremental angular velocities,

$\mathbf{T}(\Delta \alpha, \Delta \beta)$ is a transformation matrix based on the incremental incidences, 
and

$\Phi_{\mathbf{r}}$ is a rigid body modal matrix at node $n$.

On the solid surface, the incremental velocity vector is given by the incremental change of slopes $(\Delta \phi, \Delta \theta, \Delta \psi)$ arising from the elastic deformations as well as the vibration, $\dot{\eta}_{\mathbf{e}}$. i.e.

$$
\Delta \mathbf{v}=\mathbf{T}[\Delta \phi, \Delta \theta, \phi \psi] \mathbf{v}+\left[\boldsymbol{\Phi}_{\mathbf{e}}\right] \dot{\mathbf{\eta}}_{\mathbf{e}}
$$

These modifications have been implemented in the ICA-CFD code.

\subsection{Implicit acceleration scheme}

The original ICA-CFD solution algorithm has been replaced by a quasi-implicit scheme discussed in Argyris et al (1995). Equation (6) in the context of a finite element scheme may be rewritten as

$$
\mathbf{M}(\partial \mathbf{V} / \partial t)+\mathbf{R}=0
$$

where $\mathbf{M}$ is the mass-like matrix (usually a lumped diagonal matrix) and $\mathbf{R}$ is the residual flux vector. After multiplying by $\mathbf{M}^{-1}$ we get

$$
(\partial \mathbf{V} / \partial t)+\overline{\mathbf{R}}=0
$$

The normalized flux, $\overline{\mathbf{R}}$ can be expressed as

$$
\overline{\mathbf{R}}=\overline{\mathbf{R}}_{\mathbf{0}}+\left[\frac{\partial \overline{\mathbf{R}}}{\partial \mathbf{V}}\right] \Delta \mathbf{V},
$$

$(\partial \overline{\mathbf{R}} / \partial \mathbf{V})$ is a Jacobian matrix, which can be approximated as a banded matrix consisting of 3 to 9 off-diagonal elements. The elements of $(\partial \overline{\mathbf{R}} / \partial \mathbf{V})$ can be computed using the concept of system identification. The computational details may be found in Argyris et al (1995). The solution algorithm is then given by

$$
\Delta \mathbf{V}=\Delta t[\mathbf{I}+\Delta t(\partial \overline{\mathbf{R}} / \partial \mathbf{V})]^{-1} \overline{\mathbf{R}}_{0}
$$

The stability of the system of equations increases with increasing bandwidth. In the present analysis three diagonal elements were used with the global Courant-FriedrichsLewy (CFL) number $\leqslant 4 \cdot 0$. A two-step solution is recommended for nonstationary solutions.

\section{System identification}

System identification is a recursive on-line estimation procedure employed to construct equivalent mathematical models of dynamic systems using input and output sequences. This methodology has been widely applied in advanced communications, space flights and industrial automation. Here we intend to apply this scheme to determine the linearized dynamic models, $\mathbf{A}$ and $\mathbf{B}$, as proposed in $\$ 2$ (i.e. (4)).

The usual approach is to represent the differential equations in terms of $N$ previously 
known records, such as

$$
\mathbf{y}_{n-1}=\sum_{n=1}^{N}\left(\mathbf{A}_{n} \mathbf{y}_{n}+\mathbf{B}_{n} \mathbf{u}_{n}\right)
$$

and solve for the $\mathbf{A}_{n}$ and $\mathbf{B}_{n}$ matrices (Balakrishnan 1968; Hsia 1977; Åström \& Wittenmark 1989). The resulting dynamical model and the feedback gain matrix will be $N$ times larger than the original system (Pak \& Friedman 1991). This means extensive computer resources are required to design an on-line adaptive control law. To minimize the cost of computation Argyris et al (1995) employ an alternate method which requires only one sampling from an earlier record. In other words, the number of equations in the estimation process is the same as in the original model. This is a significant reduction in the overhead cost of designing a feedback control system. A brief account of this procedure is as follows:

Let

where

$$
\dot{\mathbf{x}}=\mathbf{F}^{*}(\mathbf{x}, \mathbf{u}, t)
$$

$F^{*}$ is a vector of normalized aerodynamic forces;

$\mathbf{x}$ and $\mathbf{u}$ represent the aerodynamic angles $(\alpha, \beta)$, angular velocities $(p, q, r)$

and and the control surface motion (these are the inputs to the CFD module);

$\dot{\mathbf{x}}$ represents the acceleration of the system as an output.

The desired mathematical model may then be linearized to read as

$$
\dot{\mathbf{x}}=\mathbf{A x}+\mathbf{B u}+\mathbf{f}_{0} .
$$

This equation, in the notations of autoregression scheme, may be rewritten as

where

$$
\mathbf{y}=\phi^{T} \boldsymbol{\theta},
$$

$$
\begin{aligned}
& \mathbf{y}=\dot{\mathbf{x}}^{T}, \\
& \boldsymbol{\phi}=\{\mathbf{x}, \mathbf{u}\}, \\
& \boldsymbol{\theta}=\left[\begin{array}{l}
\mathbf{A}^{T} \\
\mathbf{B}^{T}
\end{array}\right] .
\end{aligned}
$$

Since $y$ and $\phi$ are known at various time intervals, the autoregression procedure can be used to solve for $\theta$.

\section{Adaptive feedback control design}

Modern aircraft designed with relaxed static stability require digital flight control systems to provide stability and control at all flight conditions. Moreover, if the pressure distributions as computed by the CFD methods are nonlinear functions of the flight parameters then the dynamic model will be changing with time. For these reasons it is necessary that an adaptive control law based on the current estimate of aerodynamic derivatives must be implemented. In a previous section we described 
a procedure for estimating the dynamic characteristics of a system using the concept of system identification. We will now describe a design procedure which utilizes this information to design a feedback control system.

Let us consider a system of state space equations given by

$$
\dot{\mathbf{x}}=\mathbf{A x}+\mathbf{B u},
$$

and the corresponding output equations by

$$
\mathbf{z}=\mathrm{H}_{1} \mathbf{x}+\mathbf{H}_{\mathbf{2}} \dot{\mathbf{x}}=\mathbf{C x}+\mathrm{Du},
$$

where $H_{1}$ and $H_{2}$ are the output transformation matrices. The $A, B, C$ and $D$ matrices constitute what is generally known as the quadruplets of a set of state space equations. If $\mathbf{z}_{T}$ is a vector of target output of a system, the performance index function may be written as

where

$$
J=h_{T}+\int_{t}^{S_{t}}\left[\frac{1}{2} \varepsilon^{T} \mathbf{Q} \varepsilon+\frac{1}{2}\left(\mathbf{u}-\mathbf{u}_{\mathbf{c}}\right)^{T} \mathbf{R}\left(\mathbf{u}-\mathbf{u}_{\mathbf{c}}\right)+\mathbf{p}^{T}(\mathbf{F}-\dot{\mathbf{x}})\right] \mathrm{dt},
$$

$\mathbf{u}_{\mathrm{c}}$ is the pilot command,

$p$ is a vector of Lagrangian coefficients,

$\varepsilon=\left(\mathbf{z}-\mathbf{z}_{T}\right)$ is an error vector,

$\mathbf{Q}$ is a symmetric weighting matrix,

$\mathbf{R}$ is a symmetric and positive definite matrix,

and

$$
h_{T}=\frac{1}{2} \varepsilon_{T}^{T} \mathbf{Q} \varepsilon_{T} \text { is the terminal performance index at } t_{f} .
$$

The designer selects the weighting matrices, $\mathbf{Q}$ and $\mathbf{R}$, to satisfy the stability requirements. Parameters, $\mathbf{x}, \mathbf{u}$ and $\mathbf{p}$ must be determined to minimize the performance index, $J$. Hence, the variation of $J$ with respect to $x, \dot{x}, u$ and $p$, yields a system of equations for $x(t), p(t)$ and $u(t)$,

and

$$
\dot{\xi}(t)=\mathbf{H} \xi+\mathscr{T}
$$

where

$$
\mathbf{u}=-\mathscr{R}^{-1}\left[\mathbf{B}^{T} \mathbf{p}(t)+\mathbf{D}^{T} \mathbf{Q}\left(\mathbf{C x}(t)-\mathbf{z}_{T}\right)\right]
$$

$$
\begin{aligned}
\xi & =\{\mathbf{x}, \mathbf{p}\}, \\
\mathbf{H} & =\left[\begin{array}{cc}
\mathscr{A} & -\mathscr{B} \\
-\mathscr{Q} & -\mathscr{A}^{T}
\end{array}\right] \text { (Hamiltonian matrix), } \\
\mathscr{T} & =\left\{\begin{array}{l}
\mathbf{F}_{1} \\
\mathbf{F}_{2}
\end{array}\right\}
\end{aligned}
$$

in which

$$
\begin{aligned}
& \mathscr{R}=\mathbf{R}+\mathbf{D}^{T} \mathbf{Q D}, \\
& \mathscr{A}=\mathbf{A}-\mathbf{B} \mathscr{R}^{-1} \mathbf{D}^{T} \mathbf{Q C}, \\
& \mathscr{B}=\mathbf{B} \mathscr{R}^{-1} \mathbf{B}^{T}, \\
& \widetilde{\mathbf{Q}}=\mathbf{C}^{T}\left[\mathbf{Q}-\mathbf{Q D R}^{-1} \mathbf{D}^{T} \mathbf{Q}\right] \mathbf{C}, \\
& \mathbf{F}_{1}=\mathbf{B} \mathscr{R}^{-1} \mathbf{D}^{T} \mathbf{Q} \mathbf{z}_{T}, \\
& F_{2}=C^{T}\left[Q-Q D \mathscr{R}^{-1} D^{T} Q\right] \mathbf{z}_{T} \text {. }
\end{aligned}
$$


The resulting end conditions are given by

$$
\begin{aligned}
& \mathbf{x}\left(t_{0}\right)=\mathbf{x}_{0}, \\
& \mathbf{p}\left(t_{f}\right)=\left[\mathbf{C x}\left(t_{f}\right)+\mathbf{D u}\left(t_{f}\right)-\mathbf{z}_{T}\right] .
\end{aligned}
$$

This is a two-point boundary value problem. Its solution is more complicated than the usual one-point boundary value problem. A systematic solution procedure is given by Kirk (1970). For the sake of completeness we briefly summarize his procedure. The solution to (41) may be written as

$$
\xi\left(t_{f}\right)=\left[\exp \left(\mathbf{H}\left(t_{f}-t\right)\right)\right] \xi(t)+\mathbf{H}^{-1}\left[\exp \left(\mathbf{H}\left(t_{f}-t\right)\right)-\mathbf{I}\right] \mathscr{T} .
$$

Using the terminal end condition given by (53), one can solve for the Lagrangian coefficients, $\mathbf{p}$, in the following form:

$$
\mathbf{p}(t)=\mathbf{K}(t) \mathbf{x}(t)+\mathbf{z}^{\prime}(t)
$$

Substituting (55) into (42) the adaptive feedback signal reduces to

$$
\mathbf{u}=\overline{\mathscr{X}}(t) \mathbf{x}(t)+\overline{\mathbf{z}}(t)
$$

and the adaptive control law is given by $\overline{\mathscr{X}}(t)$.

If the target values, $\mathbf{z}_{T}$ were set to zero, then the above tracking problem reduces to that of a regulator. In that case $\overline{\mathbf{z}}(t)=0$.

Finally, substituting for $u$ in (36) we obtain a set of stable plant equations

$$
\dot{\mathbf{x}}=[\mathbf{A}+\mathbf{B} \overline{\mathscr{X}}(\mathbf{t})] \mathbf{x}=\overline{\mathbf{A}} \mathbf{x} .
$$

The designer may choose $\mathbf{Q}$ and $\mathbf{R}$ such that the real components of the roots of $\overline{\mathbf{A}}$ are negative (i.e., the roots lie in the left half of the s-plane).

\section{Aircraft manoeurre analysis}

In this section we specialize the equations of motion discussed in the previous sections to compute the design loads of a manoeuvring aircraft. The inertial accelerations arising from vibration are negligibly small compared to the manoeuvring accelerations and hence the acceleration terms in (2) which involve the elastic deformation terms, $\ddot{\eta}_{e}$ and $\dot{\eta}_{e}$, omitted. Then we obtain a system of equations of motion in rigid body modes including the flexibility effects of the aircraft structure. After some simplifications the final manoeuvre equation (2) reduces to the following form:

$$
\dot{\mathbf{x}}=\mathbf{F}(\mathbf{x}, \mathbf{u}, \mathbf{t}),
$$

in which the state variables are given by

$$
x=\{V, \gamma, q, \theta\},
$$

and the control variables are

$$
u=\left\{\delta_{1}, \delta_{2}, \delta T_{1}, \delta T_{2}\right\}
$$


in which $V$ is the aircraft velocity along the flight path, $\delta_{i}$ is the control surface deflection and $\delta T_{i}$ is the thrust control. The column vector $\mathbf{F}$ represents the mass normalized forces along and normal to the wind axis and the pitching moment about its mass centre. The last element in $\mathbf{F}$ is $\mathbf{F}_{4}=q=\dot{\theta}$ and represents an integrator to obtain $\theta$. The aerodynamic forces will be computed iteratively using the deformed shape of the aircraft given by

$$
\eta_{\mathrm{e}}=-\left[\mathbf{K}_{\mathrm{e}}\right]^{-1} \mathbf{F}_{\mathrm{e}} \text {. }
$$

The generalized force vector $F_{e}$ represents the net forces (aerodynamic-inertia (centrifugal)) on the aircraft.

The system of equations in (58) may be solved iteratively in the following steps:

(1) compute $\dot{\mathbf{x}}$ using initial values of $\mathbf{x}$ and $\mathbf{u}$,

(2) integrate $\dot{\mathbf{x}}$ to get $\mathbf{x}$,

(3) perform system identification to determine the state space matrices $\mathbf{A}$ and $\mathbf{B}$,

(4) compute the feedback gain matrix and the feedback signal $u$,

(5) compute the target error, $\varepsilon=\mathbf{z}-\mathbf{z}_{T}$,

(6) if $|\varepsilon|$ less than or equal to a specified value, end the computation,

(7) otherwise, repeat steps 1 through 6.

Finally, integrate the net forces to yield section design loads such as shear, bending and torsion.

\section{Discussion of results}

In the previous sections we discussed an analytical method unifying the CFD algorithm to compute flight loads and aeroelastic stability boundaries. Some preliminary test cases have been performed to validate the concept in the following areas:
(a) system identification;
(b) on-line adaptive control design;
(c) aircraft manoeuvre analysis;
(d) static aeroelastic effects and aeroservoelastic stability analysis.

\subsection{System identification and control design}

Two test cases have been selected to verify the accuracy and the reliability of these two modules. The first example represents the landing approach of the F-16 model reported by Rynaski (1982). The state variables are $\mathbf{x}=\{q, V, \alpha, \theta\}$ and the control variable is the elevator deflection $\mathbf{u}=\left\{\delta_{e}\right\}$. The corresponding state space matrices $\mathbf{A}$ and $\mathbf{B}$ are given in table 1. For a unit-step input the plant response, $\dot{\mathbf{x}}$, was computed and integrated to obtain $\mathbf{x}$. Then, the autoregression module was used to estimate the plant matrices, $\overline{\mathbf{A}}$ and $\overline{\mathbf{B}}$. Subsequently, using $\overline{\mathbf{A}}$ and $\overline{\mathbf{B}}$ the feedback control gain matrix, $\mathbf{K}$, was computed. In addition the eigenvalues of the open-loop and the closedloop plant matrices were calculated. The estimate matrices after fifty time-steps are seen to be in excellent agreement with the original data. Since the F-16 is a statically relaxed aircraft one of its roots is unstable. But, after closing the loop through the feedback control all the roots move to the left half of the s-plane. The closed-loop 


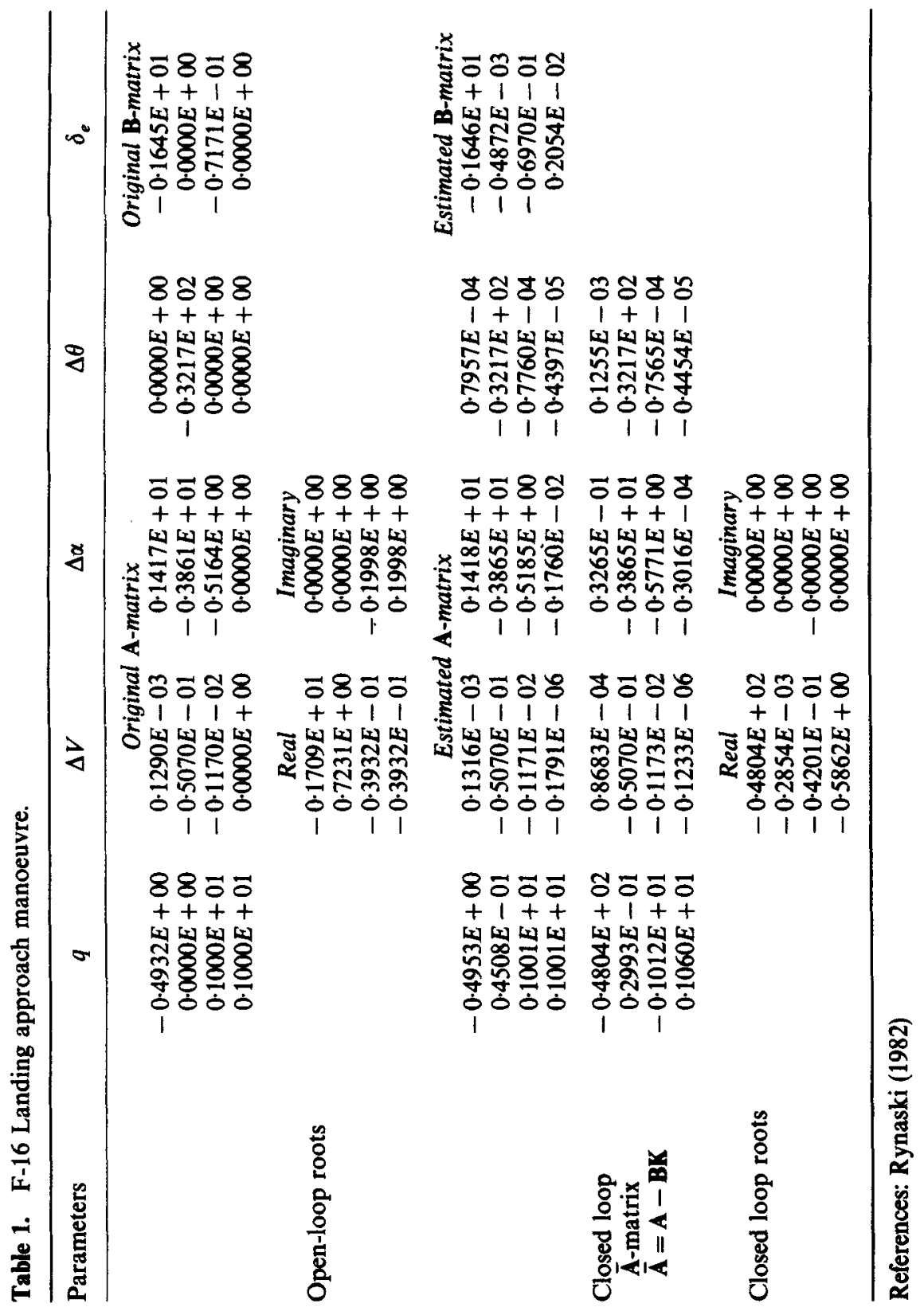



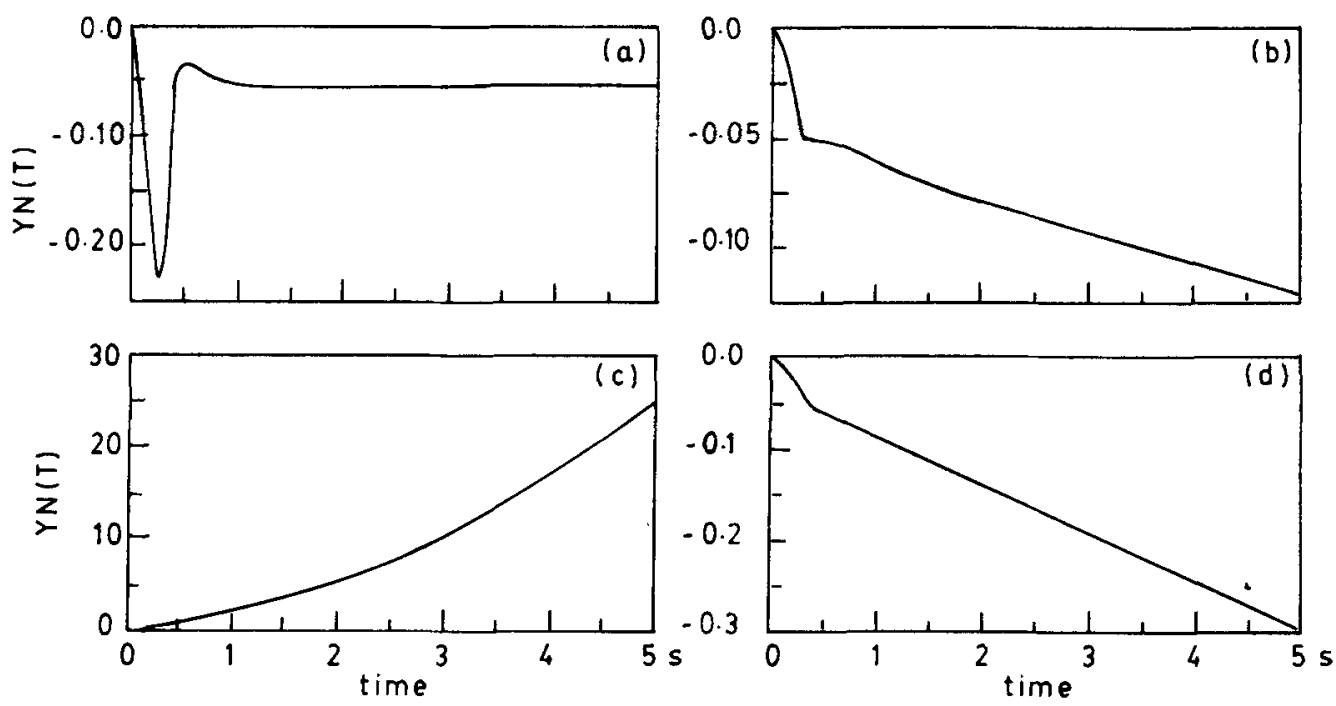

Figure 3. F-16 landing approach manoeuvre model (Rynaski 1982). (a) Pitch rate, $Q$; (b) $\alpha$; (c) speed change, $D V$; (d) pitch angle.

response characteristics of the aircraft due to a unit-step input are shown in figure 3 . The system responds very rapidly to the elevator command to gain the desired lift during a landing manoeuvre.

The second example represents the fuselage pitch pointing of the F-16 model. The state variables in this case are $\mathbf{x}=\{\theta, q, \alpha\}$, and the control variables are $\mathbf{u}=\left\{\delta_{f}\right.$ (flap), $\delta_{e}$ (elevator) $\}$. The plant matrices as reported by Porter \& Bradshaw (1981) are given in table 2. Once again the estimated plant matrices are in excellent agreement with the original data. As in the previous example the unstable roots move from the right half of the s-plane to the stable zone in the left half plane. The closed-loop response characteristics of the aircraft are shown in figure 4. Any desired response characteristics can be achieved by selecting appropriate weighting coefficients, $\mathbf{Q}$ and $\mathbf{R}$.'

These two examples and many others which are not reported here justify the use of the single sampling approach suggested in this study to estimate the plant matrices with reasonable accuracy. In addition, the on-line control design procedure used here is able to stabilize the system whose dynamic characteristics are practically unknown to begin with.

\subsection{Symmetric flight manoeuvre analysis}

To demonstrate the simulation of the manoeuvre analysis using the CFD scheme we have selected a simple delta wing with a cranked leading edge as shown in figure 5 . Two control surfaces, one inboard and one outboard, were used to trim and control the vehicle. The gross weight and the pitching moment of inertia of the vehicle were $1.55 \times 10^{6} \mathrm{~N}$ and $3.019 \times 10^{6} \mathrm{~kg} \cdot \mathrm{m}^{2}$. The centre of gravity was located at $60 \%$ of the mid chord. For 1-g level flight at Mach 0.7 , the analysis started with an initial angle to attack of $3^{\circ}$. First, 50 time-step solutions were performed just to establish the flow field around the aircraft. Then the balancing of the aircraft started with the feedback control system turned on. The state space matrices were continuously estimated 


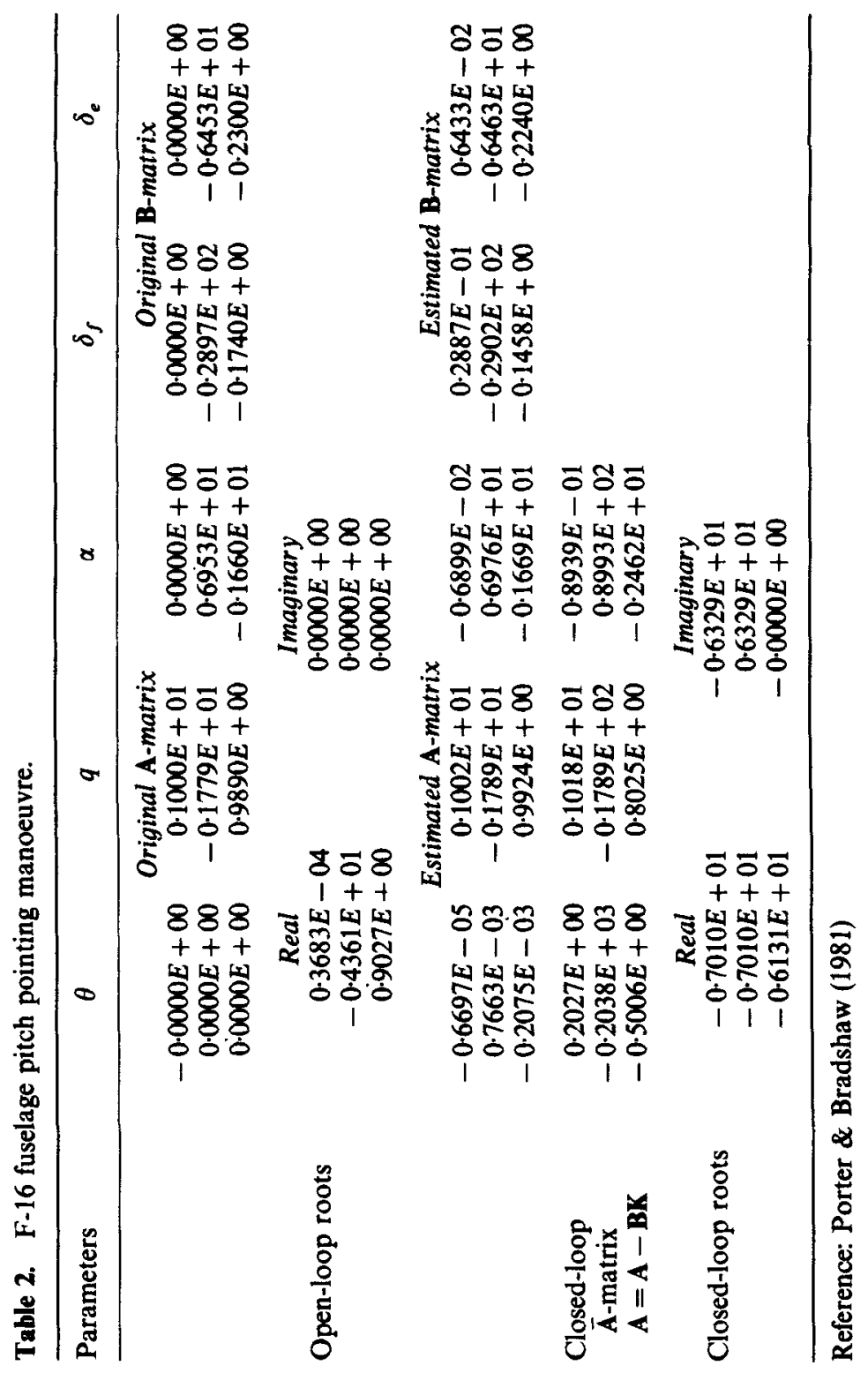



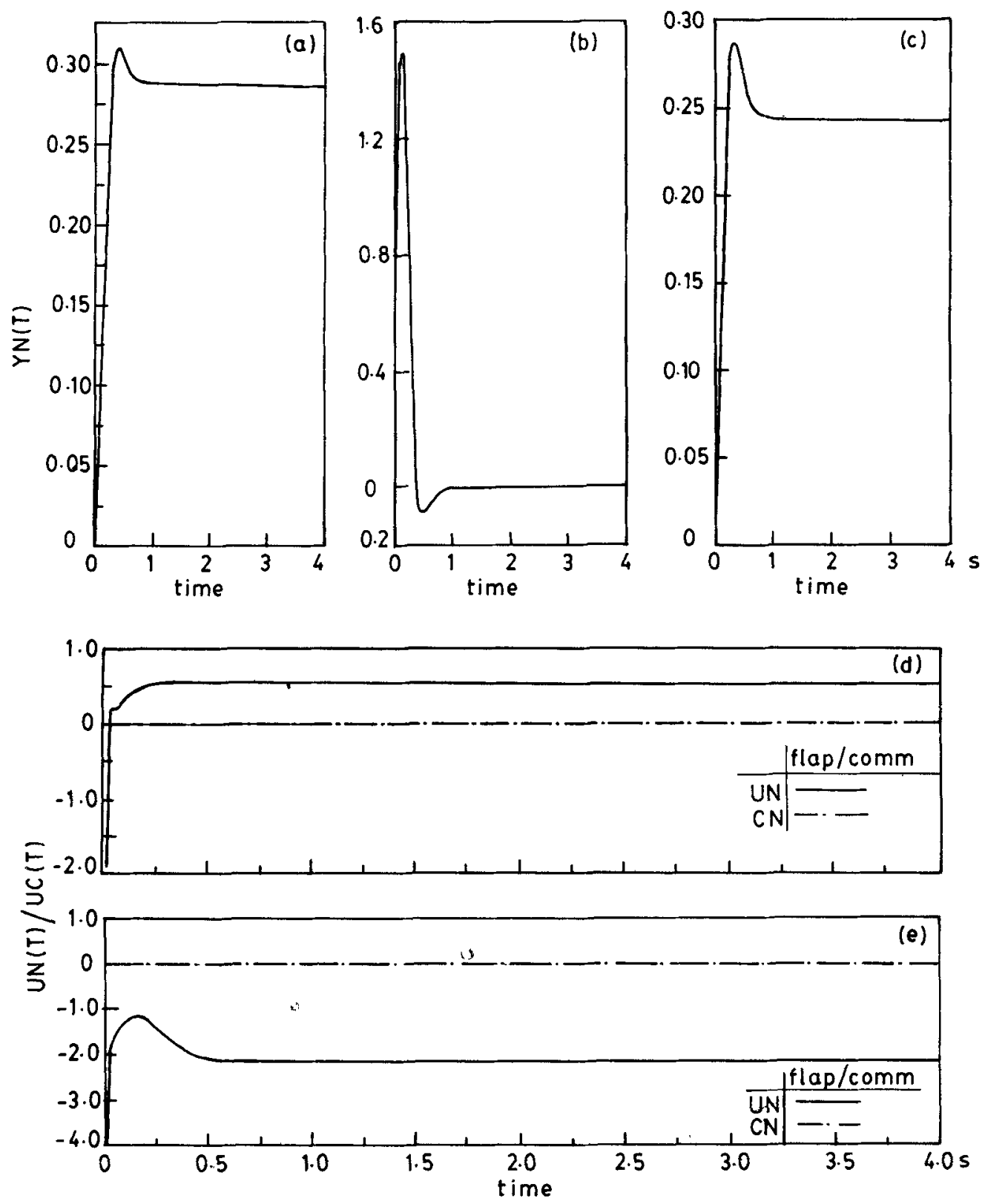

Figure 4. Fuselage pitch pointing manoeuvre model (Porter \& Bradshaw 1981):

(a) $\theta$; (b) pitch rate, $Q$; (c) $\alpha$; (d) flaperon (degrees); (e) elevator (degrees).

and the feedback gain matrices were computed. The required load factor, $n_{z}$, was used as the terminal target quantity.

The estimated plant matrices for the open-loop and the closed-loop cases are shown in table 3 . The elements of these matrices denote the stability derivatives of the aircraft with respect to $V, \gamma, q, \theta$ and $\delta$ parameters. These quantities may also be expressed 


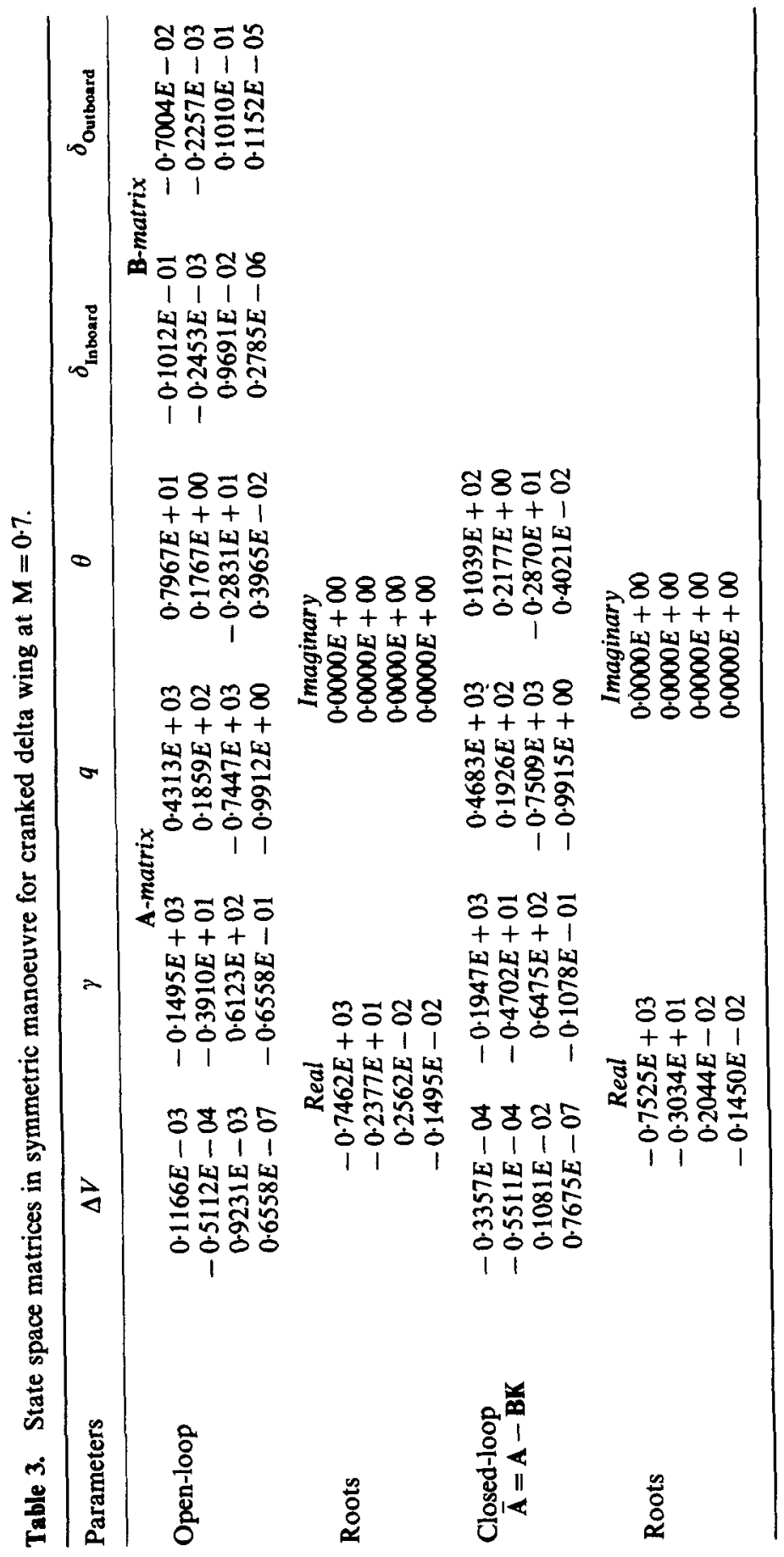




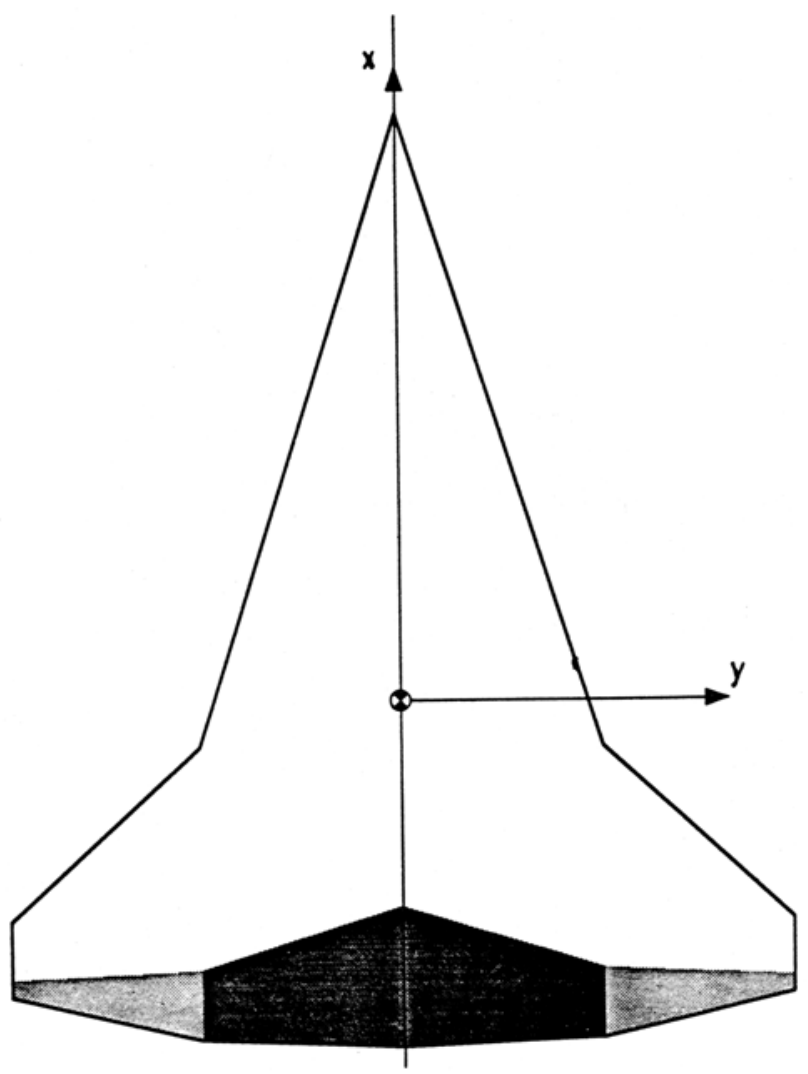

Figure 5. Cranked delta wing with inboard and outboard flaps.
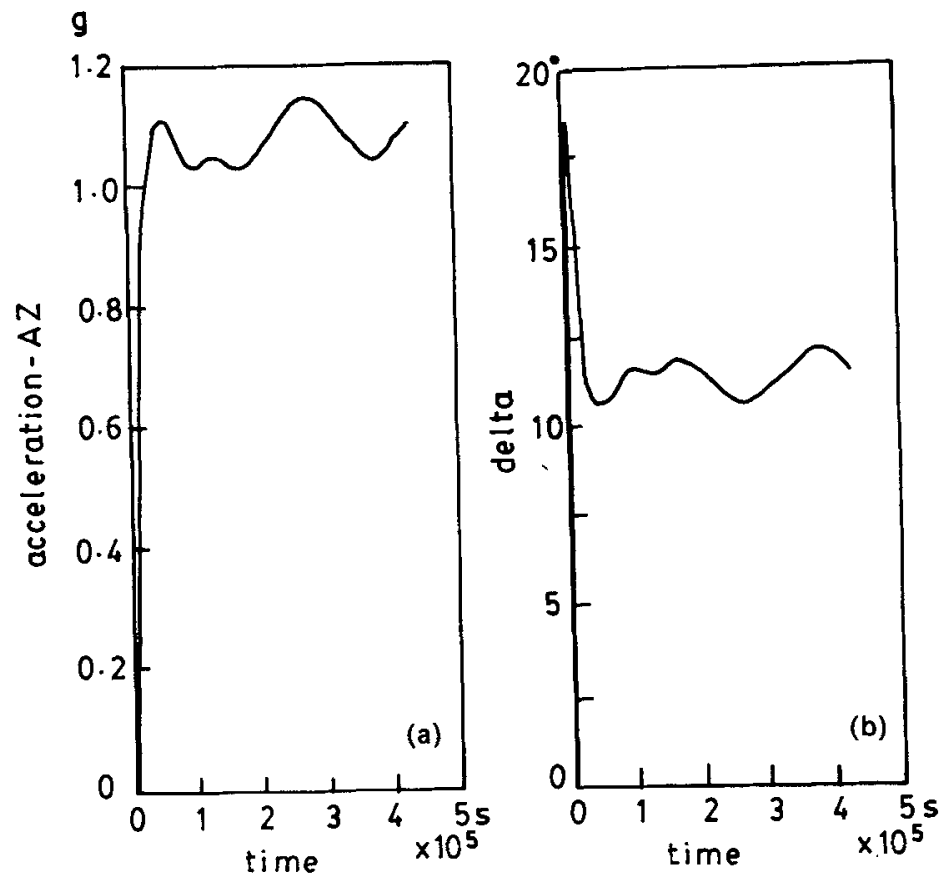

Figure 6. 1-g symmetric level flight. (a) Normal acceleration in $g$ vs time; (b) control is time. 

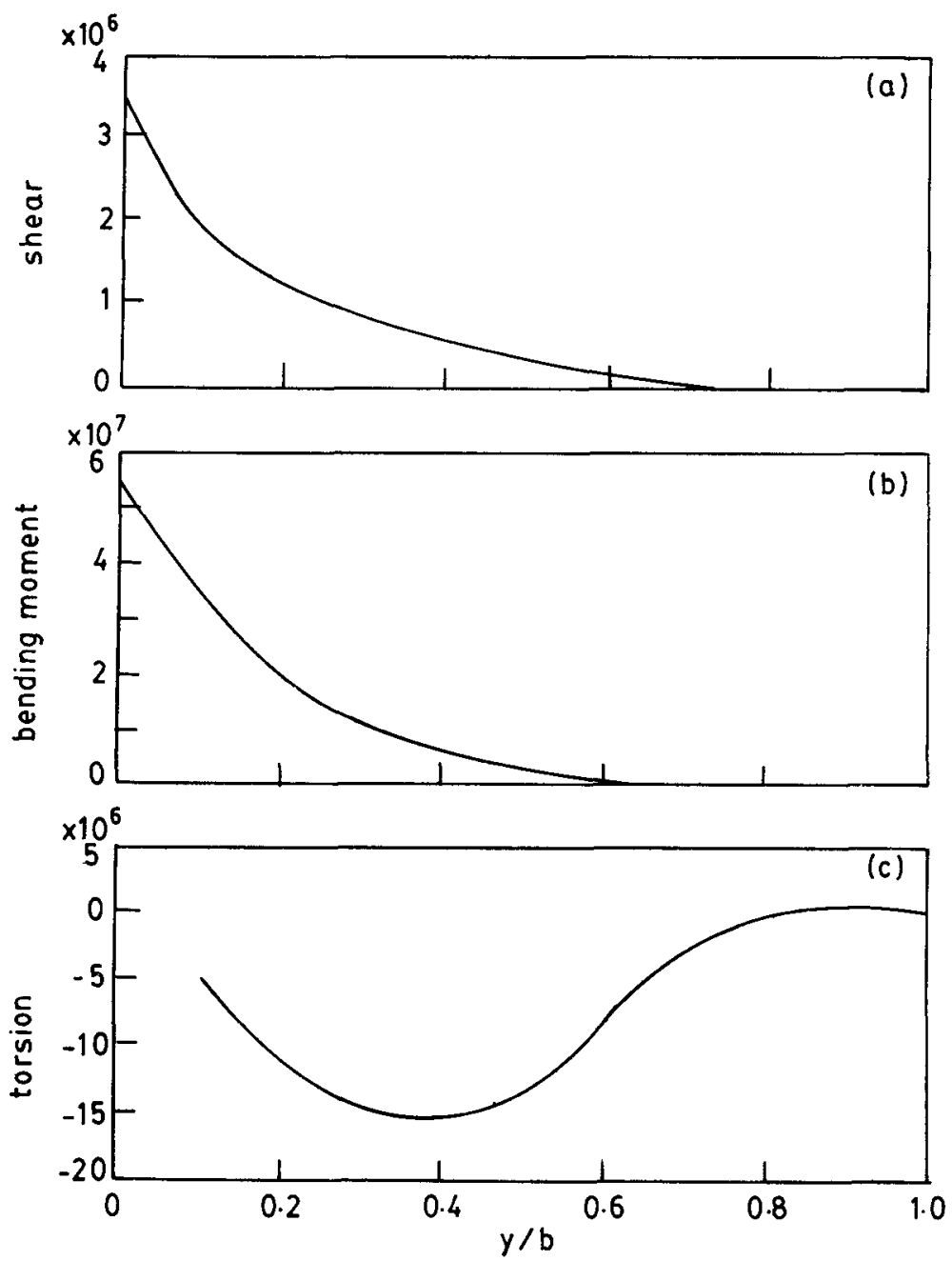

Figure 7. Integrated load distributions for $1-g$ flight at $M=0 \cdot 7$. (a) Shear; (b) bending moment; (c) torsion.

in terms of usual aerodynamic stability derivatives such as: $C_{\mathrm{L \alpha}}, C_{\mathrm{M \alpha}}, C_{\mathrm{Lq}}, C_{\mathrm{L} \delta}$ etc. for rigid as well as flexible aircraft. Figure 6 shows the time history of acceleration and the control surface rotation for $1-g$ level flight. The corresponding integrated load distributions: shear, bending moment and torsion about a reference axis at the centre of gravity are shown in figure 7. The manoeuvre data presented in this example remain to be verified with other sources for accuracy and reliability.

\section{Conclusions}

This paper summarizes a new multidisciplinary approach to computational aircraft dynamics and loads analysis. System identification has been extensively used to solve the CFD problem and to estimate the state-space matrices, which determine the stability characteristics of the system and to compute adaptive gain matrices. The 
principle of multidisciplinary analysis alluded to in this study has other promising areas for application and development. Specifically these include: (1) computation of aerodynamic stability derivatives of rigid and flexible aircraft, (2) aeroservoelastic analysis, and (3) multidisciplinary design and optimization. While the results of the specific examples presented in this study are encouraging, additional validation of the method using practical examples has yet to be addressed.

The computational effort of this project was supported by IBM of the United States of America. The authors sincerely thank Mr Raj Mantha of IBM for his active participation and interest in the progress of the project. Mr Don Rossman of IBM made necessary conversions to port the ICA-CFD code onto the IBM 3090 system. We thank him for this effort. The authors also wish to thank Messrs Juri Kalviste and Don Kesler for their valuable discussions during the development of the adaptive control laws. Finally, the authors' appreciation and thanks go to Ms R Corvese for her skillful and dedicated effort in the integration of the loads module with the CFD code.

\section{References}

Appa K 1991 Recent advances in manoeuvre loads analysis. Comput. Meth. Appl. Mech. Eng. 90: 693-717

Argyris J 1953 Thermal stress analysis and energy theorems. A. R. C 16489

Argyris J, Appa K, Bühlmeier J 1995 Texts on computational mechanics: Vol. VI. A survey of aeroservoelasticity (Amsterdam: North-Holland) (to be published)

Argyris J, Dunne P C 1947 The general theory of cylindrical and conical tubes under torsion and bending loads. J. R. Aeronaut. Soc. 51: 199-269, 757-784, 884-930

Argyris J, Dunne P C 1949 The general theory of cylindrical and conical tubes under torsion and bending loads. J. R. Aeronaut. Soc. 53: 461-483, 558-620

Argyris J, Kelsey S 1960 Part II: Energy theorems and structural analysis (London: Butterworths) (originally published in series of articles in Aircraft Engineering, Oct, Nov 1954 and Feb, March, April, May 1985)

Argyris J, Mlejnek H P 1991 Texts on computational mechanics. Vol. V. Dynamics of structures (Amsterdam: North-Holland)

Argyris J, St. Doltsinis I, Friz H 1989 Hermes space shuttle: Exploration on reentry aerodynamics. Comput. Meth. Appl. Mech. Eng. 73: 1-51

Ȧström K J, Wittenmark B 1989 Adaptive control (Addison-Wesley)

Balakrishnan A V 1968 A new computing technique in system identification, J. Computing System Sci. 2: 102-116

Hirsch C 1990 Numerical computation of internal and external flows (Chishester: John Wiley \& Sons) Vols. 1 and 2

Hsia T C 1977 System identification: Least square methods (Lexington, MA: Lexington books)

Kirk D E 1970 Optimal control theory: An introduction (Network Series) (ed.) R W Newcomb, (Englewood Cliff, NJ: Prentice-Hall)

Pak C G, Friedmann P P 1991 Transonic adaptive flutter-suppression using approximate unsteady time domain aerodynamics. Paper No. AIAA-91-0986

Porter B, Bradshaw A 1981 Design of direct digital flight mode control systems for highperformance aircraft (New York: IEEE)

Rynaski E G 1982 Flight control system design using robust output observers. Advances in Guidance and Control System: AGARD Conference proceeding, No. 321 